\title{
1 Classifying Psychopathology: Mental Kinds and Natural Kinds
}

\author{
Harold Kincaid and Jacqueline Sullivan
}

According to the Mental Health Atlas (World Health Organization 2011, p. 13), "neuropsychiatric disorders are estimated to contribute to $13 \%$ of the global burden of disease"-that is, more than 450 million people suffer from neuropsychiatric disorders. They are leading causes of disabilityadjusted life years, accounting for 37 percent of the healthy years lost from all non-communicable diseases. Depression alone accounts for one-third of this (Insel 2011).

The global cost of mental illness is estimated at 2.5 trillion US dollars and is expected to increase to more than 6 trillion dollars by 2030 (World Health Organization 2011). In the United States alone, estimates indicate that mental-health disorders account for 59 percent of the economic costs that stem from injury or illness-related loss of productivity (ibid.). Perhaps surprisingly, the economic burden of mental-health disorders stems less from the cost of care than from loss of income due to unemployment, expenses for social supports, and a range of indirect costs due to chronic disability that begins early in life (Insel 2011). According to the director of the National Institutes for Mental Health, "considering that those with mental illness are at high risk for developing cardiovascular disease, respiratory disease, and diabetes, the true costs of mental illness must be even higher" (ibid.).

While awareness of the cost of mental disorders is increasing, doubts about overmedicalization-about treating what really are problems in living as if they were medical diseases calling for pharmacological treatment-are also increasing. For example, there is evidence that certain sociological and economic pressures on the development of manuals such as the Diagnostic and Statistical Manual of Mental Disorders have eroded historically preserved distinctions between categories differentiating "normal" types of human suffering from mental disorder and dysfunction. A classic case in point, analyzed by Allan Horwitz and Jerome Wakefield 
in their 2007 book The Loss of Sadness, is the late-twentieth-century erosion of the boundaries between sadness and depression. (See chapter 11 below.) At one time, the two categories were kept distinct. However, the operationalization of depressive disorder put forward in the fourth edition of Diagnostic and Statistical Manual of Mental Disorders (referred to as DSM-IV) included conditions that served to broaden the kinds of phenomena picked out by the concept to the extent that what was previously considered sadness came to be lumped together with what was previously considered major depression. As the class of persons picked out by the category became more heterogeneous, the number of persons diagnosed as "depressed" increased, as did the number of prescriptions for anti-depressants. The removal of the grief and bereavement clause from the fifth edition of the DSM (2013) further broadens the category of major depression by allowing doctors to diagnose individuals suffering from sustained grief due to loss of a loved one as having major depression. This move is likely to impede the search for the causes of depression further and to result in an increase in the overuse of psychotropic drugs.

This volume asks whether psychiatry as a science may better position itself to cure mental-health disorders by considering whether improvements to the current criteria for classifying mental disorders are warranted or whether the classification schemes are fine as they stand. Either directly or indirectly, the authors take up the question of whether mental disorders are natural kinds. The basic idea behind the concept of natural kinds is that science identifies the most fundamental entities of nature and shows how they are interrelated. In the case of mental disorders, psychiatric categories ought to group together phenomena in such a way that those phenomena are subject to the same type of causal explanation (see, e.g., Craver 2009) and respond similarly to the same kinds of causal interventions (see, e.g., Woodward 2003). If psychiatric categories do not find such groupings, then there is reason to revise and/or eliminate existing classifications.

This volume is thus organized around the scientific ideal of natural kinds and the extent to which psychiatry can and currently does identify them. The notion of natural kinds can be read in multiple ways. It will be useful to set out some of the main variants of the concept of natural kind here.

All senses of natural kinds assume that there is something out there in the world that grounds classifications. The open questions are "What is that something like?" and "What kinds of classifications can it ground?" The strongest sense of natural kinds, often called "essentialism," thinks that there are sets of individually necessary and jointly sufficient properties 
for a given kind that entail a strict in or out classification of all individuals. The weakest sense asserts only that some properties are statistically associated; it allows for no sharp, non-arbitrary categorizing of individuals who lie on a continuum. Various other notions fall between these two poles. Pragmatic approaches might agree with the weak continuum picture but think that practical reasons such as cost effectiveness might allow for drawing motivated boundaries. The view adopted most often in this volume allows for natural kinds without a single set of necessary and sufficient conditions but instead with multiple different property combinations with family resemblance similarity relations.

These are some main contours of different views of natural kinds. There are further sophistications possible beyond these basic views, and some of them are explored in this volume. The chapters are generally united, however, in the belief that it is important for practice and scientific inquiry to get clear about what sense of natural kinds is at work in any particular classification of psychopathology.

Many of those who have participated in the natural-kinds debate about psychiatry are realists about mental disorders and would like it to be the case that psychiatric classification systems such as that of the DSM did a better job of tracking natural kinds. There are optimists; there are also pessimists. However, the validity of categories of mental disorder has never been, and continues not to be, the main aim of the task forces involved in the development of the DSM. Rather, the primary aims are intra-rater reliability and inter-rater reliability. The result is a classification system in which the necessary and sufficient conditions associated with a given category of mental disorder pick out a heterogeneous rather than a homogeneous class of individuals. In addition, boundaries between the categories fail to be robust to the extent that there is significant comorbidity between categories. These shortcomings may be regarded as rendering the classification system poorly poised to serve as a basis for identifying the causes of mental disorders and locating effective strategies for intervening in them. However, current categories are used as the primary basis for research into the causes of mental disorders, because they are the best classification schemes available. This may soon be changing, insofar as the National Institutes of Mental Health have recently "launched the Research Domain Criteria (RDoC) project to transform diagnosis by incorporating genetics, imaging, cognitive science, and other levels of information to lay the foundation for a new classification system" (Insel 2013).

Two primary themes emerge from the chapters that follow: that current classification systems of mental disorders are insufficient for the purposes 
of successful diagnosis, treatment, and research and that the assumptions on which these classification systems are based are problematic. However, the contributors provide different answers as to why the current classification systems fail, different ideas on how the failures might be overcome, and different suggestions as to how the assumptions on which the systems are based might be revised.

Nick Haslam begins his chapter by laying out the useful set of different senses of "natural kind." He argues that there probably are no cases of mental disorders that meet the strict essentialist requirements, and that different conceptions of natural kind may be appropriate for different disorders. Haslam then discusses taxometric methods for identifying natural kinds. He notes that taxometric analyses of artificial data sets with a subset of individuals that only share family resemblances conclude that there is positive evidence for a taxon. Thus, when taxometric analyses do find positive evidence for a discreet class, that evidence may be only evidence for a family-resemblance kind, not for a discrete or essentialist kind. Haslam's second point is that taxometric studies have found positive evidence for treating mental disorders as taxons in a small minority of possible disorders. Haslam concludes his chapter by describing some empirical evidence that essentialist concepts of mental disorders promote stigmatization, contrary to the widespread notion that treating mental disorders as disease will make persons diagnosed with them less prone to social discrimination.

Jeffrey Poland argues that contemporary treatment and research on mental disorders is dominated by "conventional psychiatric practice" (CPP), which is ordered around the DSM: individuals are diagnosed on the basis of the DSM, treatment and research studies are based on it, and the DSM is strongly embedded in the financial and educational sides of psychiatry. Poland claims that the empirical evidence shows that the DSM categories are a scientific failure in that none of them have been found to have predictive validity and in that they have multiple defects as a basis for treatment decisions and approaches. (See chapter 8 for a dissenting opinion.) The reason for these failures, Poland suggests, is that the assumptions of CPP and the DSM are inconsistent with the essential nature of mental phenomena and mental disorders. There is no sharp difference between normal mental functioning and the process involved in mental disorders. All mental phenomena are hierarchically organized, multidimensional, context sensitive, and so on-characteristics that don't fit well with the medical model of DSM-based practice. Unfortunately, that model is deeply embedded in current practices and institutions, and Poland 
doubts that the latest revisions in the DSM will change its basic character. Poland makes some suggestions for reform.

Dan Stein begins his chapter by pointing out that for some time it was widely believed that psychotropic drugs, insofar as they acted on different neurobiological substrates, could be used to distinguish different mental disorders from one another and even to differentiate abnormal from normal behavior. However, the fact that the action of specific psychotropic drugs is fairly diffuse and the fact that mild depression responds to placebo alone suggest that psychotropic drugs cannot be used to "carve nature at her joints" when it comes to psychiatric kinds. Stein further demonstrates the limitations of this view by pointing out that there are multiple ways to differentiate mental disorders. For example, from the "classical perspective" the correct way to individuate mental disorders is by appeal to necessary and sufficient conditions, whereas a "critical perspective" acknowledges that classification systems may change over time and may vary across cultures. In contrast, an "integrative perspective" acknowledges that, even though the categories are in part socially constructed, this does not mean that they aren't amenable to being understood scientifically and explained mechanistically, but it may indeed rule out the possibility that psychotropic agents will be sufficient for differentiating normal behavior from pathological behavior. Stein argues for this integrative perspective, and claims that it is instantiated in what he refers to as "the naturalistic approach." According to Stein, insights from cognitive psychology suggests that ordinary folk universally agree that certain kinds of phenomena may be classified as normal and others cannot be so classified. Neuroscience provides us with good reasons to believe that the mental phenomena we take to be indicative of a mental disorder correspond to or are caused by abnormal brain processes, and anthropology teaches us that social constructions influence the categories, but neither neuroscience nor anthropology rules out the possibility that psychopharmacological interventions may be used to treat the disorders corresponding to the categories. It just turns out that in instances in which a condition is deemed psychiatric yet not attributable to underlying abnormalities in the brain, and is spoken of using moral metaphors, it will probably not be subject to medical intervention, but rather to social intervention. How one regards a particular psychiatric disorder and how one thinks it is best to be treated will vary, depending on one's perspective.

Like Haslam, Peter Zachar notes that there are different senses attached to the term "natural kinds" and that the different senses may be more or less demanding. Zachar's own previous work on "practical kinds" is partial 
motivation for Haslam's framework. On Zachar's view, "being out there"not being merely a subjective fact about how we like to talk, but being something in the world-is a central feature of most notions of natural kinds. Fitting into laws, supporting induction, and identifying discrete entities are further criteria, and the strong essentialist version requires underlying causal properties that are necessary and sufficient for classification. Zachar identifies several different weaker notions of natural kinds, most of which involve some kind of objective similarity that grounds predictions. He notes that one motivation for identifying natural kinds in the study of mental disorders is scientific legitimacy. However, he denies that scientific legitimacy requires the full-fledged essentialist notion. There are useful generalizations about depression, for example, even if it is not well captured as an essentialist natural kind.

Dominic Murphy asserts at the outset of his chapter that the categories of psychiatric disorders found in the DSM are informed by folk psychology. This prompts the question of whether current taxonomic schemes in psychiatry actually track natural kinds. Specifically, Murphy has in mind Richard Boyd's (1991) view of natural kinds as homeostatic property clusters that group together phenomena that are similar enough to be subject to explanation in terms of the same underlying causal properties. In order to broach the question of whether classification systems based on folkpsychological distinctions are capable of yielding Boydian natural kinds, Murphy considers the case of delusional beliefs. The category of delusion, according to Murphy, is based on folk-epistemological considerations of what constitutes good and bad outputs of our knowledge-producing processes. While delusions are regarded as abnormal outputs, it is a separate question whether the kinds of phenomena that are grouped together under the heading of "delusion" may be used to track one or several common belief-producing mechanisms that go awry. On an optimistic interpretation of what folk-psychological thought may accomplish, we might be inclined to think that the category of delusion is an example of a Boydian homeostatic property cluster. However, on the skeptical interpretation that Murphy advocates, it is likely that the category groups together such disparate and heterogeneous phenomena that a whole host of causal processes will have to be posited in order to accommodate them. Furthermore, the same may be said for any psychiatric classification system that is informed by folk psychology.

George Graham raises the question of whether mental disorders are real. As he rightly notes, answering this question requires that we first specify what a mental disorder is, and that we then identify the evidentiary stan- 
dards required for attributing mental disorders to persons. Graham's aim is to argue for a realist position about mental disorders by answering these two questions and demonstrating that mental disorders are both "act-ofclassification-independent" and "inherent" conditions of persons. According to Graham, mental disorders are real insofar as they are psychologically incapacitated conditions in the world that inhere in persons, are independent of our actively classifying them, are harmful to the persons in whom they inhere, and are sometimes harmful to other persons. These features are true of these conditions irrespective of whether they are natural kinds or brain disorders.

Harold Kincaid defends the view that there are some categorical groupings that support objective predictive and explanatory accounts of psychopathology, though he shares the views of Haslam and Zachar that there need be no one right notion of natural kinds for psychopathology and indeed argues that many alleged psychopathologies may not be natural kinds in any sense. His notion of natural kinds is in the same genre as Boyd's concept of a homeostatic property, Haslam's discrete and prototype groups, and Zachar's variants-based ability to predict. However, he is more explicit in discussing what the ability to predict involves and in defending categorical accounts against dimensional accounts. The latter approach is favored by the dominant psychometric practice and is a serious threat to a categorical conception of natural kinds of psychopathology. Kincaid finishes by making a case that major depression is a categorical grouping that supports prediction and explanation. He agrees with Horwitz that current criteria and current screens for depression mislead, and he provides taxometric, behavioral, and neurobiological evidence that major depressive disorder constitutes a distinct set of individuals.

Nancy Nyquist Potter, Don Ross, and Allan Horwitz take up specific mental disorders and examine the complexities involved in classifying them. Potter focuses on oppositional defiant disorder and its application to African Americans, especially young men. She argues that the legacy of racism and inequality is a powerful presence in the everyday lives of African Americans, and that labeling the behavior of black boys in school as Oppositional Defiant Disorder can be regarded as a misclassification based on failure to understand the social context constituted by a society in which racism is widespread. What teachers take to be disordered defiance can be an understandable expression of self-worth in the face of preexisting racial stereotypes. Failure to show deference to teachers may be a reflection of the fact that at home teenagers and adults relate in a nondeferential way. Medical models of disorders downplay social context, 
but understanding social context is essential to differentiating real mental disorder from culturally based behavior. Like Horwitz, Potter wants sociological understanding to be used to improve classification practices. If misapplication of depression to ordinary sadness causes inappropriate treatment, so does misapplication of ODD-it may even contribute to the perpetuation of racism.

Ross surveys recent progress in delineating problem and pathological gambling. He notes at the start that this distinction is based on an analogy to the popular distinction between problem drinking and alcoholism and that how these categories are applied is a function of social norms. The task for those interested in studying and treating gambling disorders is to turn folk-psychological notions into something scientifically and clinically useful. Clinicians do not give equal weight to the various symptoms listed in the DSM, and clinical screens by design minimize false negatives and thus produce false positives. Nonetheless, different screens applied across quite different populations have reached similar conclusions about the prevalence of pathological gambling. A major open question is whether these screens are getting at qualitatively different phenomena from ordinary gambling or whether gambling problems are best regarded as situated along a continuum. (For a discussion of this issue in general for psychopathology, see chapter 8.) Evidence from neurobiology and from molecular genetics suggests that pathological gambling is a qualitatively distinct phenomenon. Those with the most serious gambling problems show disturbances in the dopamine reward system, and in the neuroadapted hypoactivity of serotonergic circuits that normally inhibit impulsive behavior, that are similar to those of drug addicts. Ross also cites the literature showing that drug addicts exhibit distinct genetic differences. One important moral is that standard screens for psychopathology are weak instruments for detecting natural kinds if they exist.

Horwitz uses a sociological understanding to point out flaws in current criteria while defending the prospect for an objective disorder. He defends the idea that depression might be a natural kind, but argues that the current DSM criteria fail to pick it out. He believes depression is a distinct naturally occurring entity because the historical record clearly identifies it across millennia. Not until the 1970s did the DSM conception take root, and the result was an explosion in the number of individuals described as suffering from depression. DSM-III put in place the current DSM depression criteria, which include a poor appetite, inability to sleep, and low energy for two weeks. The criteria are sufficiently far from serious melancholia and of such short duration that a diagnosis of major depression now comes 
much easier. Understandable reactions to life events such as job loss, divorce, and bereavement (on bereavement see DSM-5) become cases of major depression. Horwitz traces the various professional and financial interests that made the change in classification happen and sustain it today.

Şerife Tekin aims to establish that Ian Hacking's looping effects are far more complex than Hacking himself has indicated and his critics have appreciated. Tekin's primary criticism of Hacking is that although changes in an individual's "self-concept" are one of the main components of Hacking's looping effects, Hacking himself never puts forward a concept of the self (only a concept of "a classified person"), and that this has left him open to attacks by critics who argue that mental disorders are on a par with kinds in other areas of science. Tekin argues persuasively that Hacking's account of looping effects needs to be buttressed by an account of how an individual's "self-concept" changes in response to receiving a diagnosis of a mental disorder and changes further in response to the individual's experiencing himself as someone having a mental disorder. To this end, Tekin puts forward what she refers to as a model of "the multitudinous self," which she uses to provide a much more detailed understanding of the nature of Hacking's looping effects.

Jacqueline Sullivan concludes the volume with an investigation of the changes that psychiatric kinds undergo when they become explanatory targets of areas of sciences that are not "mature" (see, e.g., Hacking 1988, 1992) and are in the early stages of discovering mechanisms (see, e.g., Bechtel and Richardson 1993; Bechtel 2008; Craver 2007). She focuses on two such areas of science involved in the investigation of the mechanisms of mental disorders: cognitive neuroscience and cognitive neurobiology. Neuroscientists have recently come to understand mental disorders as disorders of cognition (see, e.g., Carter et al. 2009 and Insel 2013), and a number of intra-disciplinary and inter-disciplinary research initiatives have emerged to study their mechanisms. Sullivan evaluates one such research initiative and uses it to show that even if scientific research were to begin with somewhat "stable" psychiatric and cognitive kinds, the kinds have the potential to become wildly unstable because the areas of science studying them are relatively new and the methods put forward for individuating them are not standardized across research contexts. Sullivan argues, via an analysis of the case study, that although such instability can be ameliorated if investigators impose intra-disciplinary and inter-disciplinary "strategies of stabilization," such "unifying measures" also have certain unpalatable consequences such as potentially impeding important 
scientific discoveries. She concludes by suggesting that this tension be overcome by striking a balance between standardizing research methods and allowing for a modest pluralism.

\section{References}

Bechtel, W. 2008. Mental Mechanisms: Philosophical Perspectives on Cognitive Neuroscience. Erlbaum.

Bechtel, W., and R. Richardson. [1993] 2010. Discovering Complexity: Decomposition and Localization as Strategies in Scientific Research. MIT Press.

Boyd, R. 1991. Realism, antifoundationalism, and the enthusiasm for natural kinds. Philosophical Studies 61: 127-148.

Carter, C., J. Kerns, and J. Cohen. 2009. Cognitive neuroscience: Bridging thinking and feeling to the brain, and its implications for psychiatry. In Neurobiology of Mental Illness, third edition, ed. D. Charney and E. Nestler. Oxford University Press.

Craver, C. 2007. Explaining the Brain: Mechanisms and the Mosaic Unity of Neuroscience. Oxford University Press.

Craver, C. 2009. Mechanisms and natural kinds. Philosophical Psychology 22: $575-594$.

Hacking, I. 1988. On the stability of the laboratory sciences. Journal of Philosophy 85 (10): 507-514.

Hacking, I. 1992. The self-vindication of the laboratory sciences. In Science as Practice and Culture, ed. A. Pickering. University of Chicago Press.

Horwitz, A., and J. Wakefield. 2007. The Loss of Sadness: How Psychiatry Transformed Normal Sorrow into Depressive Disorder. Oxford University Press.

Insel, T. 2011. The global cost of mental illness. At http://www.nimh.nih.gov.

Insel, T. 2013. Transforming diagnosis. At http://www.nimh.nih.gov.

Woodward, J. 2003. Making Things Happen. Oxford University Press.

World Health Organization. 2011. Mental Health Atlas. 